LETTER TO THE EDITOR

\section{Clostridium difficile infections in a tertiary hospital: value of surveillance}

\section{Madam,}

Clostridium difficile is a Gram-positive, spore-forming anaerobic bacillus associated with antibiotic-associated diarrhoea and antibiotic-associated colitis. It is widely distributed in the environment and colonises up to $3-5 \%$ of adult humans and $84 \%$ of healthy infants without causing symptoms. ${ }^{1}$ The source of $C$. difficile may be endogenous or environmental. ${ }^{2}$ Transmission from patient to patient is via contaminated hands of healthcare workers or contaminated patient environment or equipment. ${ }^{3}$ We report our recent experiences with $C$. difficile infections at the Steve Biko Academic Hospital.

During the latter half of 2008, we observed an increase in stool specimens being submitted for routine analysis, including requests for $C$. difficile toxin. It was then decided that the data for the preceding months should be reviewed to inform us whether we were about to encounter an outbreak. In addition, we did not have an accurate knowledge of $C$. difficile infections at our hospital and the hospital did not have a written policy for the control and prevention of $C$. difficile infections.

A review of laboratory records was initially done for the period January to June 2008 because we noticed an increase in the number of stool specimens being submitted in June for the diagnosis of C. difficile infection. When an increase in the number of toxin-positive stool specimens was observed in August, we contacted the infection control nurses and planned our intervention. We drew up guidelines for the prevention and control of $C$. difficile infections, communicated these to the clinicians and hospital management staff and held training sessions with ward staff members.

The diagnosis of $C$. difficile infection was by commercial $C$. difficile toxin A test assay (Oxoid, Basingstoke, UK) which detects the presence of toxin A in stool specimens. ${ }^{4}$ Data were analysed to compare the number of requests and the results from month to month, distribution of positive results per ward and to see which hospital areas were affected.

A total of 266 stool specimens were received for the whole year and 46 specimens (17.2\%) tested positive. The 46 positive specimens were from 26 inpatients. An increase in the number of toxin positives was first seen in May, and in June the number of specimens submitted also increased (Figure 1). The months of July and August showed an increase both in specimens submitted and toxin-positive specimens (Figure 1). Therefore guidelines were drawn up and were implemented from the month of August. The subsequent months showed a plateau with some decrease in the number of toxin positives whereas December showed a very high number of requests but no real increase in the toxin-positive specimens. Infection control and prevention activities were increased in the later months of the year (August onwards). The majority of infected patients were from the surgical intensive care unit (ICU).

This study highlights the value of passive surveillance and the active participation of the infection prevention and control team. Despite the absence of firm data to support or dispute whether an outbreak was averted, the coordination between the laboratory and infection control teams was overwhelming and rewarding.

The increase in C. difficile infections in the month of August was mainly from the surgical ICU, hence the suspicion of a possible outbreak. The infections started spreading to other areas of the hospitals, namely the high dependency ward and the medical ICU. Of note is the fact that the high dependency ward is used by both the medical and the surgical ICUs as a step-down ward for recovering patients. Hence, one can speculate that the infected patients from the surgical ICU became the source for the new infections that spread to other areas of the hospital.

Most of the literature is about $C$. difficile outbreaks and there are limited data on routine surveillance for $C$. difficile. ${ }^{5}$ It is for this reason that according to our opinion we probably successfully prevented or managed an outbreak very early on.

The co-ordination between the laboratory staff, infection prevention and control team, and the different heads of clinical disciplines including the hospital management contributed in preventing further spread of this infection. This is interesting from the South African perspective as there are no data on the prevalence of C. difficile in hospitals and no screening prior to admission to hospitals. Awareness of $C$. difficile infections in our hospital continued through circulated reports via different departments. An infection prevention control policy was developed for our institution based on published guidelines. 6,7

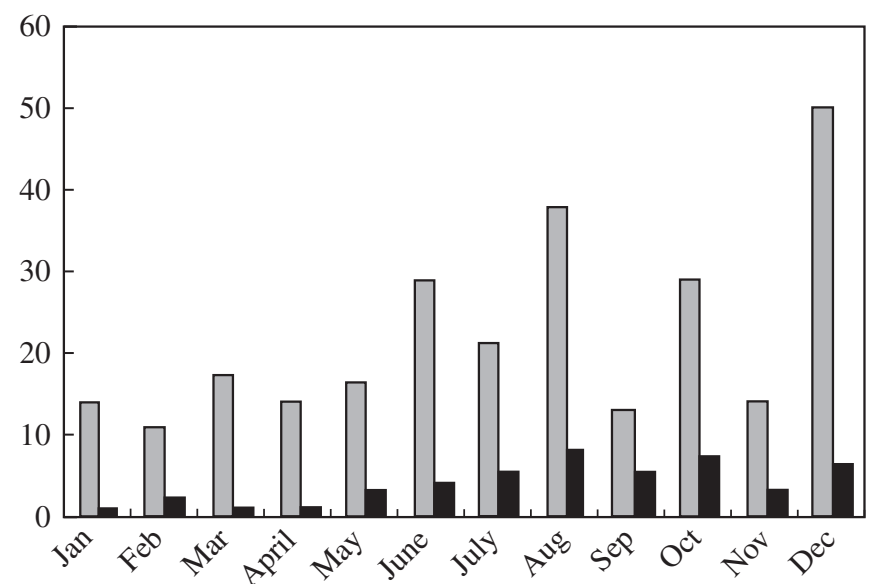

Figure 1. Number of specimens submitted and number positive for toxin according to months $(N=266)$. Grey bars: number of requests; black bars: positives. 


\section{Conflict of interest statement}

None declared.

\section{Funding sources}

None.

\section{References}

1. Bouza E, Munoz P, Alonso R. Clinical manifestations, treatment and control of infections caused by Clostridium difficile. Clin Microbiol Infect 2005;11:57-64.

2. Hanna P. Clostridium difficile is no longer just a nosocomial infection or an infection of adults. Int J Antimicrob Agents 2009;33(Suppl. 1):42-45.

3. Mutters R, Nonnenmacher C, Susin C, Albrecht U, Kropatsch R, Schumacher S. Quantitative detection of Clostridium difficile in hospital environmental samples by real time polymerase chain reaction. J Hosp Infect 2009;71:43-48.

4. Fitzpatrick F, Oza A, Gilleece A, O'Byrne AM, Drudy D. Laboratory diagnosis of Clostridium difficile associated disease in the Republic of Ireland: a survey of Irish microbiology laboratories on behalf of the $C$. difficile subcommittee of the Health Protection Surveillance Centre. J Hosp Infect 2008;68:315-321.

5. Gerding DN, Muto CA, Owens RCJ. Measures to control and prevent Clostridium difficile infection. Clin Infect Dis 2008;46:43-49.

6. Wilcox MH, Fawley WN, Wigglesworth N, Parnell P, Verity P, Freeman J. Comparison of the effect of detergent versus hypochlorite cleaning on environmental contamination and incidence of Clostridium difficile infection. J Hosp Infect 2003;54:109-114.
7. Bala H. Contamination, disinfection, and cross-colonization: are hospital surfaces reservoirs for nosocomial infection? Clin Infect Dis 2004;39: $1182-1189$.

M.R. Lekalakala ${ }^{\mathrm{a}, *}$

E. Lewis ${ }^{\mathrm{b}}$

A.A. Hoosen ${ }^{a}$

${ }^{a}$ Department of Medical Microbiology, Faculty of Health Sciences, University of Pretoria \& Microbiology Laboratory, Tshwane Academic Division, National Health Laboratory Service (NHLS), South Africa

${ }^{\mathrm{b}}$ Infection Control and Prevention Division, Steve Biko Academic Hospital, Pretoria, South Africa

* Corresponding author. Address: Department of Medical Microbiology, Faculty of Health Sciences, University of Pretoria \& Microbiology Laboratory, Tshwane Academic Division, National Health Laboratory Service (NHLS), Pretoria, Gauteng 0200, South Africa. Tel.: +27 823750685; fax: +27 123219456 .

E-mail address: ruth.lekalakala@up.ac.za 